\title{
A machine learning-based classification approach on Parkinson's disease diffusion tensor imaging datasets
}

\author{
Jannik Prasuhn ${ }^{1,2}$, Marcus Heldmann ${ }^{2,3}$, Thomas F. Münte ${ }^{2}$ and Norbert Brüggemann ${ }^{1,2^{*}}$ (D)
}

\begin{abstract}
Introduction: The presence of motor signs and symptoms in Parkinson's disease (PD) is the result of a long-lasting prodromal phase with an advancing neurodegenerative process. The identification of PD patients in an early phase is, however, crucial for developing disease-modifying drugs. The objective of our study is to investigate whether Diffusion Tensor Imaging (DTI) of the Substantia nigra (SN) analyzed by machine learning algorithms (ML) can be used to identify PD patients.
\end{abstract}

Methods: Our study proposes the use of computer-aided algorithms and a highly reproducible approach (in contrast to manually SN segmentation) to increase the reliability and accuracy of DTI metrics used for classification.

Results: The results of our study do not confirm the feasibility of the DTI approach, neither on a whole-brain level, ROI-labelled analyses, nor when focusing on the SN only.

Conclusions: Our study did not provide any evidence to support the hypothesis that DTI-based analysis, in particular of the SN, could be used to identify PD patients correctly.

Keywords: Parkinson's disease, DTI, Machine learning, Substantia nigra, Neuroimaging

\section{Background}

Diffusion tensor imaging (DTI) has been proposed for analyzing microstructural integrity not only of white but also grey matter. However, the use of DTI to observe, e. g., subcortical grey matter changes is currently under debate [15]. Whether microstructural alterations of the whole brain, regions of interests (ROI)-labeled grey matter, or the substantia nigra (SN) can be detected applying diffusion metrics in Parkinson's disease patients (PD) is still unclear. The significance of several previous DTI studies in PD is limited due to small sample sizes and by the fact that specific regions of interests were delineated manually for the extraction of diffusion metrics. Besides,

\footnotetext{
*Correspondence: norbert.brueggemann@neuro.uni-luebeck.de

1 Department of Neurology, Institute of Neurogenetics, University of Lübeck,

Ratzeburger Allee 160, 23538 Lübeck, Germany

²Department of Neurology, University Medical Center Schleswig-Holstein,

Campus Lübeck, Ratzeburger Allee 160, 23538 Lübeck, Germany

Full list of author information is available at the end of the article
}

studies that were able to demonstrate significant group differences have also shown a relevant overlap of diffusion metrics between PD patients and healthy controls, which undermines the potential diagnostic use. Machine learning-based (ML) models might help to detect subtle alterations of diffusion metrics, by their multivariate nature and by the integration of different imaging modalities, and to improve their diagnostic use subsequently. The aforementioned practice also hindered the translation into clinical practice [19]. Our study hypothesizes that ML algorithms and the application of a suitable sub-cortical atlas for the elderly population can be used to distinguish between PD patients and age- and gendermatched healthy controls in a standardized and therefore potentially more sensitive manner [5]. Computing algorithms like binary support vector machines (bSVM) or multiple-kernel learning (MKL) provide suitable and promising tools to address classification problems based on neuroimaging data [18]. Advancements in the

(c) The Author(s). 2020 Open Access This article is licensed under a Creative Commons Attribution 4.0 International License, which permits use, sharing, adaptation, distribution and reproduction in any medium or format, as long as you give appropriate credit to the original author(s) and the source, provide a link to the Creative Commons licence, and indicate if changes were made. The images or other third party material in this article are included in the article's Creative Commons licence, unless indicated otherwise in a credit line to the material. If material is not included in the article's Creative Commons licence and your intended use is not permitted by statutory regulation or exceeds the permitted use, you will need to obtain permission directly from the copyright holder. To view a copy of this licence, visit http://creativecommons.org/licenses/by/4.0/. 
multivariate interpretation of neuroimaging data have already been proven useful in a plethora of neuropsychiatric [16] and neurodegenerative diseases [11, 12]. Besides, the employment of machine-learning algorithms to Parkinson's disease datasets has offered unique advancements in interpreting distinct neuroimaging modalities [3, 4, 20, 23]. MKL also yields the opportunity to concatenate different imaging modalities. This is of particular interest as distinct diffusion metrics are meant to resemble different histopathological hallmarks of neurodegeneration [22].

\section{Methods}

DTI datasets of 162 PD patients (age: $63.9 \pm 9.3$ years; gender: $34.2 \%$ female; disease duration: $6.5 \pm 4.1$ months; mean MDS-UPDRS-III: $13.9 \pm 2.1$; mean Hoehn and Yahr stadium: $1.2 \pm 0.3$ ) and 70 age and gender-matched healthy controls $(\mathrm{HC})$ (age: $62.1 \pm 10.1$ years; gender: $34.9 \%$ female) were analyzed. This study used human subject recordings chosen from the Parkinson's Progression Marker Initiative (PPMI) database. The PPMI dataset was published open-access with a positive ethics statement of the responsible authorities. Therefore, additional ethics committee approvals do not apply to this study. DTI-MR sequences were acquired on a Siemens $3 \mathrm{~T}$ TIM Trio scanner using a 12-channel matrix head coil and a two-dimensional echo-planar DTI sequence $\left(\mathrm{TR} / \mathrm{TE}=900 / 88 \mathrm{~ms}\right.$, flip angle $=90^{\circ}$, voxel size $=2 \times 2 \times$ $2 \mathrm{~mm}^{3}, 72$ slices, 64 gradient directions with a b-value of $\left.1000 \mathrm{~s} / \mathrm{mm}^{2}\right)$. In addition, a non-gradient volume $(b=0$ $\mathrm{s} / \mathrm{mm}^{2}$ ) was acquired as well. Further details of the PPMI image acquisition protocol can be seen online (http://www.ppmi-info.org/wp-content/uploads/2017/ 06/PPMI-MRI-Operations-Manual-V7.pdf). We performed pre-processing by using the PANDA-toolbox (v1.3.1) in Matlab 2018b, including normalization to standard space (via FMRIB58_FA template, $2 \mathrm{~mm} \times 2$ $\mathrm{mm} \times 2 \mathrm{~mm}$ voxel size) [6]. In addition to conventional diffusion metrics (FA, MD, AD, and RD), we calculated local diffusion homogeneity (LDH) as another measure of microstructural white matter integrity. For the interpretation of DTI images, we calculated the following standard diffusion metrics based on the threedimensional diffusion of water as a function of spatial location: Fractional Anisotropy (FA) is a summary measure for interpreting microstructural integrity. Mean Diffusivity (MD) is a measure of the cell membrane density. It is, therefore, sensitive for cellularity, edema, and necrosis of investigated tissue. Axial Diffusivity (AD) decreases in axonal injury. Radial Diffusivity (RD) increases in de- or dysmyelination of axons. A concise review article on the interpretability of diffusion metrics to investigate microstructural grey and white matter changes are described in a review article by Alexander et al. [1]. Local diffusion homogeneity (LDH) is another diffusion metric that is specifically relevant to assess tissue homogeneity based on neighboring voxels [9]. We computed LDH for 6, 18, and 26 neighboring voxels using Spearman's Rank Correlation coefficient (06LDHs, 18LDHs, and 26LDHs) and Kendall's coefficient concordance (06LDHk, 18LDHk, and 26LDHk) [9]. Voxelwise whole-brain analysis was performed using the FMRIB58_FA template. We performed ROI-labeled analyses based on the well-established AAL atlas [21]. To further increase the signal-to-noise ratio, we additionally performed classification after masking of the SN using the ATAG atlas for the elderly population [10]. The datasets were classified through bSVMs (for single modalities) as well as MKL (for concatenated modalities). Ten-fold cross-validation (CV) and nested (leave one subject out) hyperparameter optimization as implemented in the PRoNTo-Toolbox (v2.1) [18]. The determination of relevant bSVM and MKL parameters (such as the applied L1 regularization method or the nested hyperparameter optimization) is following standard practice and is extensively described in the publications of Schrouff et al. [17, 18]. Age, gender, and total intracranial volume were used as covariates. Balanced Accuracy (BA) and area under the curve of the receiver-operating characteristic curve (ROC-AUC) were calculated to assess classification performance and were compared to random permutation testing (against 10.000 permutations).

\section{Results}

The application of the bSVM on the various types of diffusion metrics revealed that there are no significant differences concerning the BA or the ROC-AUC for voxel-wise whole-brain or AAL-based ROI-labeled analyses (data not shown here). As most studies suggest, diffusion metrics are most likely altered in the $\mathrm{SN}$ of PD patients, making the SN the region of highest interest to increase the signal-to-noise ratio for classification [19]. Therefore, further analyses focused on the diffusion metrics of the masked $\mathrm{SN}$ and will be reported in the following (see Fig. 1). Again, there were no significant differences regarding $B A$ or ROCAUC: FA (BA: 47.83\% ROC-AUC: 0.42); MD: (BA: $50.00 \%$, ROC-AUC: 0.54); AD: (BA: $50.00 \%$, ROCAUC: 0.44$) ; \quad$ RD: (BA: 50.00\%, ROC-AUC: 0.56); 06LDHs: (BA: 49.47\%, ROC-AUC: 0.54); 18LDHs (BA: 56.64\%, ROC-AUC: 0.57); 26LDHs (BA: 53.14\%, ROCAUC: 0.53); (BA: 55.03\%, ROC-AUC: 0.58); 06LDHk (BA: 55.25\%, ROC-AUC: 0.52); 18LDHk (BA: 53.14\%, ROC-AUC: 0.53); 26LDHk (BA: 51.80\%, ROC-AUC: $0.52)$. The results also indicate that the concatenation of diffusion metrics via MKL did not add any relevant information to improve the overall classification performance: $\mathrm{FA}+\mathrm{MD}+\mathrm{AD}+\mathrm{RD} \quad$ (BA: 49.44\%, ROC- 


\section{Receiver Operating Characteristics Curves}
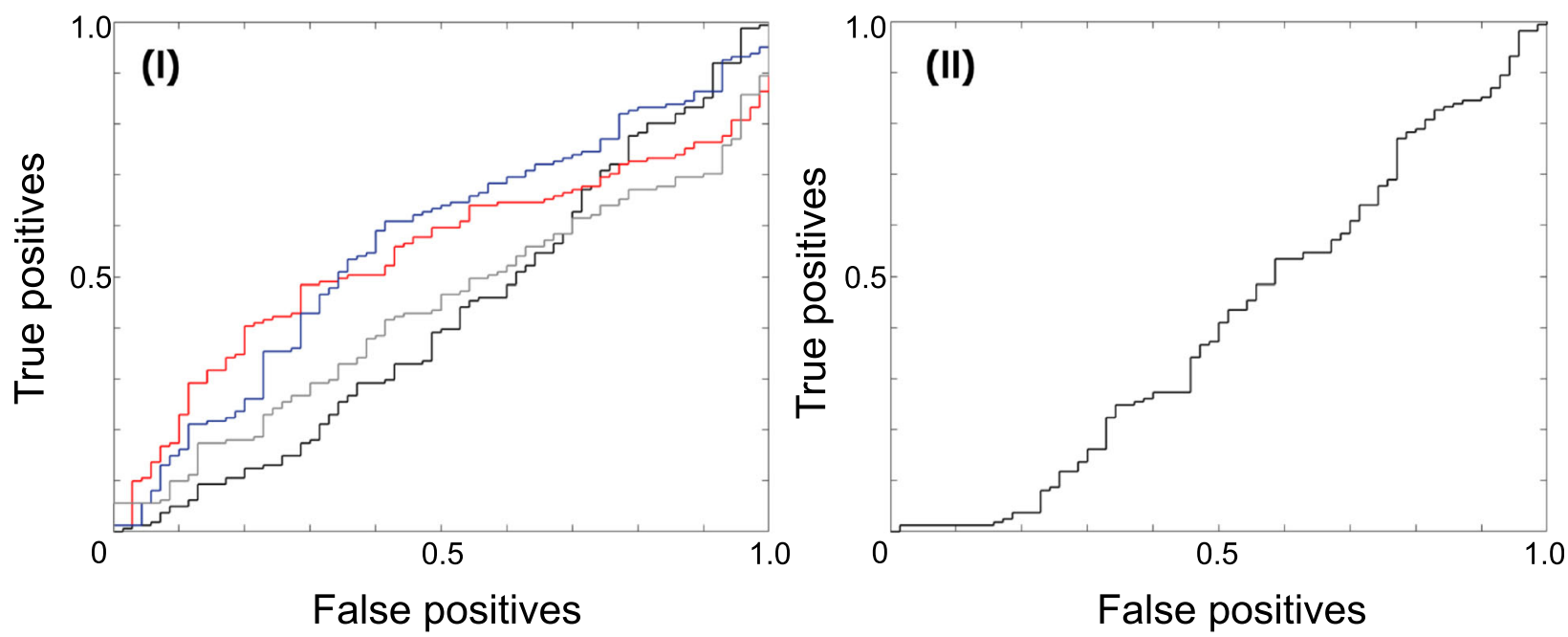

Fig. 1 ROCs for (I) FA (red), MD (black), RD (blue), and AD (grey) each as a single modality (bSVM) and (II) as concatenated modalities (MKL). Based on our results, the ROCs are indicating no substantial diagnostic value. Further, the concatenation of DTI modalities yields no additional information for this classification problem

AUC: 0.41$) ; \quad 06 \mathrm{LDHs}+18 \mathrm{LDHs}+26 \mathrm{LDHs} \quad$ (BA: $56.15 \%$, ROC-AUC: 0.60$) ; \quad 06 \mathrm{LDHK}+18 \mathrm{LDHK}+$ 26LDHk (BA: 58.12\%, ROC-AUC: 0.52). An overview on provided diagnostic performances in displayed in the Table 1 . The comparison to random permutation testing showed that the classifications, as mentioned above, did not outperform pure chance. Additionally, calculated weight maps are indicating a random weighting distribution of voxels within the $\mathrm{SN}$ used for the respective classifications (see Fig. 2), which is in contrast to previously reported changes of the dorsolateral portion of the SN (i. e., the nigrosome-1) [13].

\section{Discussion}

In this study, we demonstrated a standardized and systematic approach to potentially attain the individual discrimination of PD patients from healthy controls using DTI datasets. This approach comprised the preprocessing of the data, the automatized selection of appropriate features, and the subsequent classification. Atkinson-Clement, Pinto, Eusebio, and Coulon [2] already stated that "[...] they did not observe a PD induced reduction of nigral FA" but also that "this observation is in contrast with some recent publications claiming very high diagnostic accuracy, but [are] well in line with other reports showing small or no PD induced

Table 1 Overview of diagnostic performances of single modalities (bSVM) and concatenated modalities (MKL) for the SN

\begin{tabular}{|c|c|c|c|c|c|c|c|c|c|}
\hline \multicolumn{5}{|l|}{ bSVM } & \multicolumn{5}{|l|}{ MKL } \\
\hline & BA [\%] & ROC-AUC & Sens [\%] & $\overline{\text { Spec }[\%]}$ & & BA [\%] & ROC-AUC & Sens [\%] & Spec [\%] \\
\hline$F A$ & 47.8 & .42 & 47 & 48 & $F A+M D+A D+R D$ & 49.4 & 41 & 44 & 60 \\
\hline$M D$ & 50.0 & .54 & 55 & 42 & $06 \mathrm{LDHs}+18 \mathrm{LDH} s+26 \mathrm{LDHs}$ & 56.1 & 60 & 54 & 56 \\
\hline$A D$ & 50.0 & .44 & 40 & 47 & $06 L D H k+18 L D H k+26 L D H k$ & 58.1 & 52 & 56 & 41 \\
\hline$R D$ & 50.0 & .54 & 48 & 41 & & & & & \\
\hline O6LDHs & 49.4 & .54 & 40 & 44 & & & & & \\
\hline $18 L D H s$ & 56.6 & .57 & 49 & 41 & & & & & \\
\hline $26 L D H s$ & 53.1 & .53 & 51 & 60 & & & & & \\
\hline O6LDHK & 55.2 & .52 & 51 & 56 & & & & & \\
\hline $18 L D H K$ & 53.1 & .53 & 60 & 63 & & & & & \\
\hline
\end{tabular}

Besides BA and ROC-AUC. Sens and Spec are listed to enhance the transparency of reported ROC-AUC results

$A D$ Axial diffusivity, BA Balanced accuracy, bSVM Binary Support vector machine, FA Fractional anisotropy, $L D H$ Local diffusion homogeneity, MD Mean diffusivity, MKL Multiple-kernel learning, RD Radial diffusivity, ROC-AUC Receiver operator characteristics area under the curve, Sens Sensitivity, Spec Specificity, SN Substantia nigra 

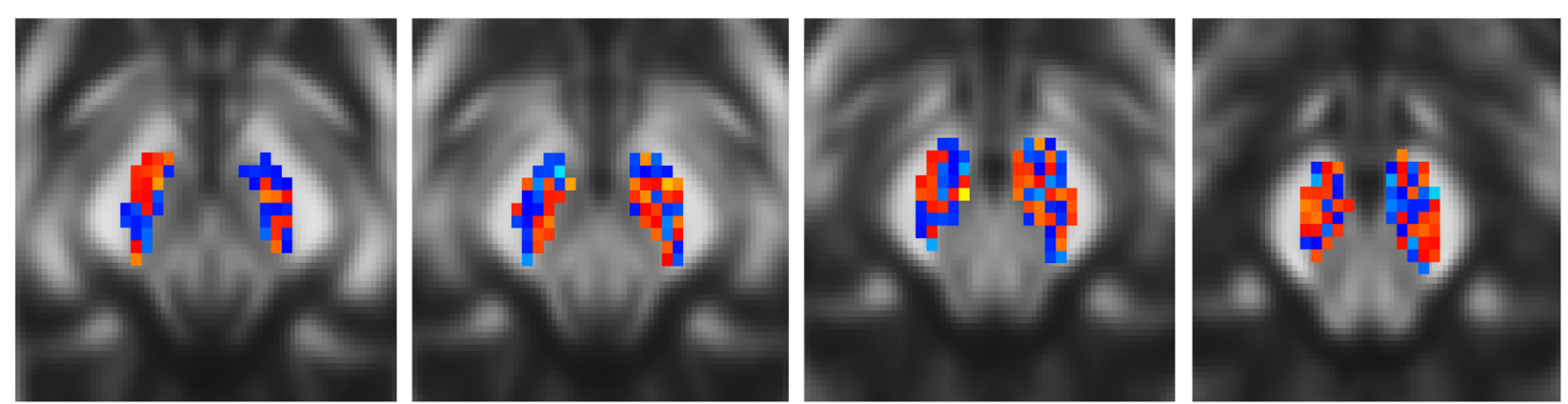

Fig. 2 Weight maps of FA bSVM (shown on axial midbrain slices). The pattern indicates random weighting of FA values for the purpose of classification (comparable to the other investigated diffusion metrics and the MKL, data not shown here). Former studies demonstrated altered diffusion metrics in the occipital portions of the SN [13]. However, interpretability is limited e.g. due to the small ROI size

nigral FA decrease". A meta-analysis also did "not support nigral DTI metrics as a useful diagnostic marker of PD" [19]. Our results are supporting the aforementioned lack of evidence and should put discussions about the diagnostic use of diffusion metrics in PD patients to rest. The negative results of our study most likely reflect the lacking suitability of diffusion metrics to investigate $\mathrm{SN}$ related microstructural alterations in PD. The interpretation of our findings within the scope of differing DTI acquisition schemes and MRI scanner hardware is challenging. However, a multicenter validation study by the authors of Fox et al. [7] stated high intersiteconcordance for applied DTI metrics on different scanner hardware (3 $\mathrm{T}$ magnetic field strength). MLalgorithms are a more standardizable and sensitive method to increase diagnostic accuracy and to disentangle the overlap of diffusion metrics other groups reported, which were only using voxel-wise massunivariate or manually extracted diffusion metrics for subsequent analysis. The multivariate, compared to mass-univariate, approach and the additional concatenation of modalities should enhance the discriminatory, and therefore, diagnostic accuracy substantially. The lack of significant findings despite a larger sample size and a more sensitive and sophisticated approach in this study are further supporting the view that traditional diffusion metrics are indeed missing any diagnostic use. Whether DTI can be used to map individual disease progression remains, to this point, elusive. Further methodological improvements of diffusion-based imaging might improve diagnostic accuracy and might, therefore, cause a reconsideration of our current conclusion. However, the current MRI acquisition and analysis paradigms of DTI measures are not of any use for investigating grey matter alterations in PD. Further studies without substantial methodological improvements will most likely not result in potentially translatable advancements in improving diagnostic accuracy or patient care. Recent research studies which revealed that the use of free-water corrected diffusion maps for the analysis of tissue alterations might provide the opportunity for fostering the diagnostic accuracy based on this dataset [14]. However, ML analyses of neuroimaging data is a fruitful approach in supporting clinical decision making and will be more frequently applied in the future [8]. The objective of our study was to investigate the role of ML-based algorithms on diffusion metrics to identify PD patients correctly. Our study did not provide any evidence to support the hypothesis that DTI-based analysis, in particular of the $\mathrm{SN}$, could be used to resolve the issue of correctly classifying study participants independent of the phenotype. An advantage of our methodology is that by calculating weighting maps, we can additionally validate our findings: Previous literature stated that the dorsolateral parts of the SN are the ones that are particularly affected at the beginning of the disease [19]. Weighting maps should indicate the higher relevance of these specific areas for classification performance (which is in contrast to our findings, see Fig. 2). Here, this advantage is of even higher importance as further partitioning of the $\mathrm{SN}$ appears, within the scope of the already small region and the present image resolution, not to be feasible.

\section{Conclusion}

Our findings are well in line with previous publications using conventional analyses. Further studies without substantial methodological improvements (e. g., utilizing more complex diffusion models) will most likely not result in potentially translatable advancements in improving diagnostic accuracy or patient care.

\section{Abbreviations}

AAL: Automated Anatomic Labeling; AD: Axial diffusivity; ATAG: Atlas of the basal ganglia; BA: Balanced accuracy; bSVM: Binary support vector machine; CV: Cross-validation; DTI: Diffusion tensor imaging; FA: Fractional anisotropy; HC: Healthy controls; LDH: Local diffusion homogeneity; MD: Mean diffusivity; MKL: Multiple-kernel learning; ML: Machine learning; MRI: Magnetic Resonance Imaging; n/a: Not applicable; PD: Parkinson's disease; PPMI: Parkinson's Progression Marker Initiative; RD: Radial diffusivity; ROC- 
AUC: Receiver operator characteristics area under the curve; ROI: Region of interest; SN: Substantia nigra

\section{Acknowledgements}

The authors would like to gratefully thank the initiators of the PPMI study, the executing staff, and the enrolled participants.

\section{Authors' contributions}

JP conceptualized and executed this research project, designed and performed the statistical analyses, and wrote the first draft. $\mathrm{MH}$ provided review and critique for the statistical analyses and the manuscript. TFM provided review and critique for the statistical analyses and the manuscript. NB conceptualized this research project, designed and reviewed the statistical analyses, and reviewed the manuscript. The author(s) read and approved the final manuscript.

\section{Funding}

JP received funding from the Parkinson's Foundation (Miami, Florida, USA), the Deutsche Parkinsongesellschaft (Berlin, Germany), and the Deutsche Forschungsgemeinschaft via the Clinician Scientist School Lübeck (DFG-GEPR IS 413535489). PPMI, a public-private partnership, is funded by the Michael J. Fox Foundation for Parkinson's Research and funding partners, as are listed at http://www.ppmi-info.org/about-ppmi/who-we-are/study-sponsors.

\section{Availability of data and materials}

The proposed study has been performed on publicly-available data obtained from the Parkinson's Progression Marker Initiative (PPMI). Study analyses were performed following the PPMI Data Use Agreement (http://www.ppmi-info. org/documents/ppmi-data-use-agreement.pdf)

\section{Ethics approval and consent to participate}

Data used in the preparation of this article were obtained from the Parkinson's Progression Markers Initiative (PPMI) database (www.ppmi-info. org/data). Ethics approval and informed written consent of all study participants has been provided by PPMI. For up-to-date information on the study, visit www.ppmi-info.org.

\section{Consent for publication}

N/A.

\section{Competing interests}

The authors have no competing or conflicting interest to report.

\section{Author details}

'Department of Neurology, Institute of Neurogenetics, University of Lübeck, Ratzeburger Allee 160, 23538 Lübeck, Germany. 'Department of Neurology, University Medical Center Schleswig-Holstein, Campus Lübeck, Ratzeburger Allee 160, 23538 Lübeck, Germany. ${ }^{3}$ Institute of Psychology II, University of Lübeck, Ratzeburger Allee 160, 23538 Lübeck, Germany.

Received: 20 July 2020 Accepted: 26 October 2020

Published online: 10 November 2020

\section{References}

1. Alexander, A. L., Lee, J. E., Lazar, M., \& Field, A. S. (2007). Diffusion tensor imaging of the brain. Neurotherapeutics, 4(3), 316-329.

2. Atkinson-Clement, C., Pinto, S., Eusebio, A., \& Coulon, O. (2017). Diffusion tensor imaging in Parkinson's disease: review and meta-analysis. Neuroimage: Clinical, 16, 98-110.

3. Ballarini, T., Mueller, K., Albrecht, F., Růžička, F., Bezdicek, O., Růžička, E., ... Schroeter, M. L. (2019). Regional gray matter changes and age predict individual treatment response in Parkinson's disease. Neurolmage: Clinical, 21, 101636.

4. Cherubini, A., Nisticó, R., Novellino, F., Salsone, M., Nigro, S., Donzuso, G., \& Quattrone, A. (2014). Magnetic resonance support vector machine discriminates essential tremor with rest tremor from tremor-dominant Parkinson disease. Movement Disorders, 29(9), 1216-1219.

5. Coutanche, M. N., Thompson-Schill, S. L., \& Schultz, R. T. (2011). Multi-voxel pattern analysis of $\mathrm{fMRI}$ data predicts clinical symptom severity. Neuroimage, 57(1), 113-123.
6. Cui, Z., Zhong, S., Xu, P., He, Y., \& Gong, G. (2013). PANDA: A pipeline toolbox for analyzing brain diffusion images. Frontiers in Human Neuroscience, $7,42$.

7. Fox, R. J., Sakaie, K., Lee, J. C., Debbins, J. P., Liu, Y., Arnold, D. L., ... Fisher, E. (2012). A validation study of multicenter diffusion tensor imaging: Reliability of fractional anisotropy and diffusivity values. American Journal of Neuroradiology, 33(4), 695-700.

8. Fu, C. H. Y., \& Costafreda, S. G. (2013). Neuroimaging-based biomarkers in psychiatry: Clinical opportunities of a paradigm shift. The Canadian Journal of Psychiatry, 58, 499-508.

9. Gong, G. (2013). Local diffusion homogeneity (LDH): An inter-voxel diffusion MRI metric for assessing inter-subject white matter variability. PLoS One, 8(6), e66366.

10. Keuken, M. C., Bazin, P. L., Schafer, A., Neumann, J., Turner, R., \& Forstmann, B. U. (2013). Ultra-high 7T MRI of structural age-related changes of the subthalamic nucleus. Journal of Neuroscience, 33, 4896-4900.

11. Khedher, L., Ramírez, J., Górriz, J. M., Brahim, A., Segovia, F., \& Alzheimer's Disease Neuroimaging Initiative (2015). Early diagnosis of Alzheimer's disease based on partial least squares, principal component analysis and support vector machine using segmented MRI images. Neurocomputing, 151, 139-150.

12. Magnin, B., Mesrob, L., Kinkingnéhun, S., Pélégrini-Issac, M., Colliot, O. Sarazin, M., ... Benali, H. (2009). Support vector machine-based classification of Alzheimer's disease from whole-brain anatomical MRI. Neuroradiology, 51(2), 73-83.

13. Menke, R. A., Scholz, J., Miller, K. L., Deoni, S., Jbabdi, S., Matthews, P. M., \& Zarei, M. (2009). MRI characteristics of the substantia nigra in Parkinson's disease: A combined quantitative T1 and DTI study. Neuroimage, 47(2), 435441.

14. Pasternak, O., Sochen, N., Gur, Y., Intrator, N., \& Assaf, Y. (2009). Free water elimination and mapping from diffusion MRI. Magnetic Resonance in Medicine, 62, 717-730.

15. Rulseh, A. M., Keller, J., Tintěra, J., Koží̌̌ek, M., \& Vymazal, J. (2013). Chasing shadows: What determines DTI metrics in gray matter regions? An in vitro and in vivo study. Journal of Magnetic Resonance Imaging, 38, 1103-1110.

16. Sacchet, M. D., Prasad, G., Foland-Ross, L. C., Thompson, P. M., \& Gotlib, I. H. (2015). Support vector machine classification of major depressive disorder using diffusion-weighted neuroimaging and graph theory. Frontiers in Psychiatry, 6, 21

17. Schrouff, J., Rosa, M. J., Rondina, J. M., Marquand, A. F., Chu, C., Ashburner, J., ... Mourão-Miranda, J. (2013). PRoNTo: Pattern recognition for neuroimaging toolbox. Neuroinformatics, 11(3), 319-337.

18. Schrouff, J., Monteiro, J. M., Portugal, L., Rosa, M. J., Phillips, C., \& MourãoMiranda, J. (2018). Embedding anatomical or functional knowledge in whole-brain multiple kernel learning models. Neuroinformatics, 16(1), $117-$ 143.

19. Schwarz, S. T., Abaei, M., Gontu, V., Morgan, P. S., Bajaj, N., \& Auer, D. P. (2013). Diffusion tensor imaging of nigral degeneration in Parkinson's disease: A region-of-interest and voxel-based study at $3 T$ and systematic review with meta-analysis. Neuroimage Clinical, 3, 481-488.

20. Segovia, F., Illán, I. A., Górriz, J. M., Ramírez, J., Rominger, A., \& Levin, J. (2015) Distinguishing Parkinson's disease from atypical parkinsonian syndromes using PET data and a computer system based on support vector machines and Bayesian networks. Frontiers in Computational Neuroscience, 9, 137.

21. Tzourio-Mazoyer, N., Landeau, B., Papathanassiou, D., Crivello, F., Etard, O., Delcroix, N., ... Joliot, M. (2002). Automated anatomical labeling of activations in SPM using a macroscopic anatomical parcellation of the MNI MRI single-subject brain. Neuroimage, 15(1), 273-289.

22. Wei, P., Leong, D., Calabrese, E., White, L., Pierce, T., Platt, S., \& Provenzale, J. (2013). Diffusion tensor imaging of neural tissue organization: Correlations between radiologic and histologic parameters. The Neuroradiology Journal, 26, 501-510.

23. Wu, Y., Jiang, J. H., Chen, L., Lu, J. Y., Ge, J. J., Liu, F. T., .. Wang, J. (2019). Use of radiomic features and support vector machine to distinguish Parkinson's disease cases from normal controls. Annals of Translational Medicine, 7(23), 773.

\section{Publisher's Note}

Springer Nature remains neutral with regard to jurisdictional claims in published maps and institutional affiliations. 